\title{
Diagnostic value of musculoskeletal ultrasound in patients with low back pain - a review of the literature
}

\author{
Plamen Todorov $^{1}$, Rodina Nestorova ${ }^{2}$, Anastas Batalov $^{1}$
}

${ }^{1}$ Medical University of Plovdiv, Rheumatology Clinic, Kaspela University Hospital, Plovdiv, ${ }^{2}$ St Irina Rheumatology Centre, Sofia, Bulgaria

\begin{abstract}
Patients with low back pain (LBP) frequently undergo various imaging studies in the pursuit of a more precise diagnosis. Ultrasound (US) has the advantage of being a widely available, multiplanar, fast and radiation-free diagnostic tool. Moreover, compared to most of the other imaging modalities, it is particularly efficient in the visualization and assessment of soft tissues. Consequently, the question about the possible diagnostic application of US in such a common pathology as LBP is very relevant to the clinical practice. For this reason, we performed a review of the literature on the diagnostic value of US in different conditions that could cause LBP. We hereby discuss available studies on the diagnostic application of US in spinal canal stenosis and disc herniation (probably of historical significance only), as well as in the pathology of soft tissue structures like the lumbar and pelvic ligaments, muscles and entheses, the thoracolumbar fascia and the sacroiliac joints (maybe of greater importance nowadays). The evidence for the diagnostic value of US is not equivocal, though promising for some of the causative conditions, and clearly this area remains open to further research.
\end{abstract}

Keywords: low back pain; etiology; ultrasound

Low back pain (LBP) occurs in various groups of the population, affects men and women equally, and is among the major reasons for rheumatological or orthopedic consultations. Its prevalence increases steadily with age and the rate of recurrence within one year could reach $44 \%$ [1]. Many imaging modalities are available to clinicians for evaluating LBP. The application of these modalities depends mainly on the working diagnosis, the urgency of the clinical problem, the availability, and the comorbidities of the patient [2]. Conventional radiography $(\mathrm{CR})$ and computerized tomography $(\mathrm{CT})$ are associated with radiation exposure and show primarily the bony elements of the lower back. Their widespread use in the 20th century might be among the reasons why paraspinal

Received 28.07.2017 Accepted 27.09.2017

Med Ultrason

2018, Vol. 20, No 1, 80-87

Corresponding author: Plamen Todorov

Rheumatology Clinic, Kaspela University

Hospital, block 2,

64 Sofia street, Plovdiv 4002, Bulgaria

Phone: +359888566478

E-mail: drtodorovplamen@gmail.com soft tissues have somehow been neglected as a cause for LBP [1]. Magnetic resonance imaging (MRI) shows both bony and soft tissue structures in the axial skeleton, but its use is hampered by the duration of the examination, its relatively high costs, its limited availability (mainly in the tertiary centers) and the contraindications for this diagnostic modality [2].

Musculoskeletal ultrasound (US) is a safe, fast, inexpensive, and widely available imaging modality that is very well tolerated by patients [3]. It allows multiplanar and dynamic examinations of the musculoskeletal system and can show the soft tissues in great anatomical detail. US is used by a growing number of physicians and the list of its applications in rheumatology and orthopedics is growing [4].

Therefore, the question of the possible diagnostic application of US in such a common condition as LBP is very relevant to the clinical practice. On one hand, this could decrease the radiation exposure associated with $\mathrm{CR}$ and $\mathrm{CT}$, and reduce the costs paid for MRI. The effect could be even bigger in time, as LBP is frequently a chronic or recurrent disease [1]. On the other hand, by 
means of US, the perispinal/perisacral soft tissues, not well visible with $\mathrm{CR}$ and $\mathrm{CT}$, could be evaluated in greater detail. For this reason, we made an appraisal of the diagnostic value of US in different conditions that could cause LBP, based on the available literature. We found that contrary to the well-established role of US in guiding the interventions in the spine, its application as a diagnostic method in LBP is still rather controversial. The difficulties in establishing US diagnostic value in LBP are further augmented by the fact that various structures could be a source or a cause of LBP [5]. For some of them there are studies in the literature, while for others such data is lacking (table I). Consequently, we hereby review the use of US as an imaging modality in the diagnosis of discrete conditions and anatomical structures that could cause LBP, rather than in LBP in general.

\section{US and the bony structures of the lumbar spine}

\section{Spinal canal stenosis}

In the 1970s, Porter et al were the first to use US as a diagnostic tool in LBP, measuring the diameter of the lumbar spinal canal for bony stenosis. They placed the transducer in an oblique midsagittal plane, $1 \mathrm{~cm}$ lateral to the midline at the $\mathrm{L} 5$ to visualize the spinal canal at this level [6]. The average diameter of the canal in patients with LBP was $1.44 \mathrm{~cm}$, while that of healthy controls was $1.61 \mathrm{~cm}$. This difference was found to be statistically significant. In addition, a cutoff value of $1.4 \mathrm{~cm}$ was proposed, with a lower diameter of the spinal canal putting the subject in the risk category for nerve roots compression and pain due to spinal stenosis. In a later study, Porter et al also published results on the spinal canal diameter measurements by the same technique in more than 700 subjects from infancy to the age of 65 years, showing that the canal was relatively wide in children, reached its maximum diameter in the late teens, and reduced slightly by late adult life [7]. Later, in a study of hospital employees an odd ratio of 10.7 was calculated for taking a sick leave due to LBP, and the spinal canal size being less than the tenth percentile of the measurements [8]. At the same time, other authors had similar results in a population of mining employees [9]. This technique was even considered as a pre-employment screening test for manual workers [9]. Other studies however concluded that the sonographic measurement of the spinal canal diameter by dorsal approach was operator dependent [10] and lacked reliability in elderly people [11].

Table I. Proposed sources and causes of LBP (based on [5]) and the presence of corresponding US studies.

\begin{tabular}{|c|c|c|}
\hline Structure or cause & US study [references] & Comments \\
\hline \multicolumn{3}{|l|}{ Bony structures: } \\
\hline Vertebral bodies & No & \\
\hline Kissing spines & No & \\
\hline Lamina impaction & No & \\
\hline Spondylolisis & No & \\
\hline Spinal canal stenosis & Yes [6-13] & Conflicting data, only historical significance. \\
\hline \multicolumn{3}{|l|}{ Intervertebral discs: } \\
\hline \multicolumn{3}{|l|}{ Joints: } \\
\hline Zygapophysial (facet) joints & No & \\
\hline SIJs & Yes [18-33] & $\begin{array}{l}\text { Some promising results in sacroiliitis and SIJD, no data on } \\
\text { other possible causes for SIJ pain. }\end{array}$ \\
\hline \multicolumn{3}{|l|}{ Soft tissues and others: } \\
\hline Thoracolumbar fascia & Yes $[35,36]$ & US can show structural changes in the fascia layers. \\
\hline Fat herniation & No & \\
\hline Compartment syndrome & No & \\
\hline \multicolumn{3}{|l|}{ Muscles: } \\
\hline Muscle spasm & Yes $[37,39,40,42]$ & US can show muscle contractures and morphologic features. \\
\hline Muscle imbalance & Yes $[37,39,40]$ & \\
\hline Muscle sprain & No & \\
\hline Muscle trigger points & No & \\
\hline \multicolumn{3}{|l|}{ Ligaments: } \\
\hline PSL & Yes $[44,45]$ & US shows spinal and posterior pelvic ligaments in sufficient \\
\hline ILL & Yes [46] & for their assessment details. \\
\hline PLC & Yes [47] & \\
\hline Iliac crest pain syndrome & Yes [49] & US can detect enthesopathies at the iliac crest as a probable cause. \\
\hline Dural pain & No & \\
\hline Epidural plexus & No & \\
\hline
\end{tabular}

SIJ: sacroiliac joints; SIJD: sacroiliac joints dysfunction; PSL: posterior sacroiliac ligament; ILL: iliolumbar ligament; PLC: posterior ligament complex. 
For this reason, Tervonen et al measured the spinal canal diameter through a transabdominal acoustic window [12]. Sensitivity of $84 \%$ and specificity of $60 \%$ were reported, when again a cutoff point of $1.4 \mathrm{~cm}$ was used.

The measurement of the spinal canal diameter by US, however, never gained widespread acceptance and in a later 10-year prospective study, Porter et al concluded that the spinal canal size on its own, had no reliable role as a predictor or an estimator of the prognosis in LBP [13].

\section{US and the intervertebral discs}

\section{Internal disc disruption (degeneration) and disc herniation}

In 1989, Merx et al compared the results of transabdominal US examination of the lumbar discs in 50 patients with discogenic LBP to the results of lumbar myelography and CT [14]. The sonographic examination was inconclusive in $18 \%$ of the patients. The study revealed a sensitivity varying from $63 \%$ to $77 \%$ depending on the disc level. Comparing these results to lumbar myelography and CT scanning, the authors concluded that US should not be used as a diagnostic modality in disc degeneration disease.

At the same time Kamei et al evaluated the effectiveness of transabdominal US to diagnose herniated nucleus pulposus in 80 patients [15]. Herniation was surgically confirmed in 41 discs. Of these $78 \%$ (32 discs) were with true-positive ultrasound diagnoses, 90\% (37 discs) with true-positive myelographic diagnoses, and 50\% (20 discs) with true-positive neurologic diagnoses. Thus, US proved to be of added value to the neurological examination, but inferior to myelography.

In 1991 Tervonen et al performed a study in which transabdominal US was compared to CT/discography [16]. They examined a total of 56 discs in 29 patients. The sensitivity of US to recognize a discographically painful disc was $95 \%$, but its specificity was only $38 \%$. US examination of lumbar discs was considered suitable only as a screening tool prior to CT/discography.

The largest study on detecting disc herniation by means of transabdominal US scanning was performed by Berth et al in 2003 on 119 patients with discogenic LBP [17]. The diagnostic accuracy of US was compared to MRI as a gold standard. It depended on the level of the disc, with sensitivity and specificity being as follows: $46 \%$ and $91 \%$ at the $\mathrm{L} 3 / 4$ level; $83 \%$ and $65 \%$ at the $\mathrm{L} 4 / 5$ level; $78 \%$ and $67 \%$ at the $\mathrm{L} 5 / \mathrm{S} 1$ level. These results confirmed the previous observations that the diagnostic performance of US in intervertebral disc herniation depend on the lumbar level.
Generally, US was found to be inferior to MRI, CT, discography, and myelography in detecting lumbar disc degeneration or herniation. Nonetheless, most of the authors concluded that transabdominal US could be used as a screening examination to reduce the radiation exposure or painful interventions to a certain lumbar level.

\section{US and the sacroiliac joint}

The sacroiliac joints (SIJ) could be affected by various conditions and are thought to account for up to $20 \%$ of all cases of chronic LBP [5].

\section{SIJ dysfunction}

In SIJ dysfunction the pain is considered to be caused by too lax or too stiff joints. In 1995 Buyruk et al developed a noninvasive technique, called Doppler imaging of vibration (DIV) to measure the laxity of the SIJ in an objective and repeatable way [18]. They used a special device to apply vibrations to the anterior iliac spines of subjects in supine position. The vibrations were registered with an US transducer placed over the ipsilateral SIJ and processed in color Doppler US (CDUS) mode. The intensity of the vibrations was measured indirectly in threshold units (TU). A large difference in the TU's values between the sacral and the iliac bones (across the SIJ) indicated a lax joint, while conversely the absence of a difference pointed to a stiff joint. The results in healthy volunteers showed satisfactory interindividual reproducibility and no significant differences between the right and the left SIJ [19]. Damen et al applied this technique in women with and without pregnancy related posterior pelvic pain [20]. The difference in the laxity between the left and the right SIJ was significantly higher (2.2 TU) in women with pain, compared to women without pain $(0.9 \mathrm{TU})$. This asymmetric laxity correlated with higher values of pain measured on the Visual Analogue Scale (VAS) and a higher number of positive pain provocation tests. Later, the same group found that a difference of more than 3.0 TU in the laxity of the left and the right SIJ had a sensitivity of $65 \%$, a specificity of $83 \%$, and a positive predictive value of $77 \%$ for the pregnancy related posterior pelvic pain to persist after the delivery [21].

Regardless of these promising results, a later review concluded that a thorough study of the validity of the technique was still missing and so the conclusions from DIV measurement should be drown with caution [22].

\section{Inflammation of the SIJ}

Spadaro et al used grey scale US to search for an effusion in the SIJ in 45 spondyloarthritis $(\mathrm{SpA})$ patients and 30 controls, and compared the findings to the results of a set of clinical tests designed to assess the SIJ [23]. The transducer was placed transversely over the SIJ. They 
found an effusion in $38.9 \%$ of the patients and only in $1.7 \%$ of the controls. The positive likelihood ratio (LR) for the presence of inflammatory LBP was 2.67 for the patients with an effusion. This result was higher than the positive LR of any single clinical test and equal to the positive LR of a combination of three tests being positive $(\mathrm{LR}=2.88)$. The authors used standard US equipment with a $7.5 \mathrm{MHz}$ linear transducer thus pointing to the applicability of this diagnostic modality in the general rheumatological practice.

Other researchers however used more sophisticated methods to detect SIJ inflammation. In their first study on contrast-enhanced US (CEUS), Klauser et al showed that the administration of intravenous contrast media improved the sensitivity from $18 \%$ to $94 \%$ and the negative predictive value from $72 \%$ to $94 \%$ for sacroiliitis [24]. However, the first-generation contrast media which was used had shorter contrast duration and could lead to overestimation of contrast enhancement caused by early bubbles distraction. In a later study, the same group used second-generation microbubbles contrast agents for the detection of active sacroiliitis. They measured the depth of the contrast US enhancement in the dorsocaudal part of the SIJ in 42 patients and 21 controls [25]. CEUS showed enhancement depth into the joint cleft of $18.5 \mathrm{~mm}$ (range $16-22.1 \mathrm{~mm}$ ) in all clinically active SIJs, significantly deeper compared to both inactive joints of patients (3.6 $\mathrm{mm}$, range $0-12 \mathrm{~mm})$, and the healthy controls $(3.1 \mathrm{~mm}$, range 0-7.8 mm). The agreement between CEUS and the clinical rating of SIJ was excellent (100\%), both positive and negative predictive values being $100 \%$ in the detection of clinically active sacroiliitis. Moreover, all inactive joints were correctly classified based on the lack of deep enhancement. The authors pointed out that while vascularization around the dorsal superficial SIJ could be seen in healthy subjects, the extension of this vascularity into the deeper parts of the joints was found only in symptomatic patients. As the mean examination time was only 11 minutes and the cost was less than one third of that for contrast-enhanced MRI, the authors believed that CEUS of the SIJs might be a cost-effective first imaging study in patients with inflammatory LBP. However, this modality could encounter difficulties when the joints are too narrow or with many osteophytes, as well as in patients with increased Body Mass Index (BMI). In addition, the application of the contrast itself has a list of contraindications [25].

Chronologically, however, the first study that assessed the possible application of US in the diagnosis of SIJ inflammation, used color Doppler US [26]. This study found surprisingly high sensitivity of CDUS $(100 \%)$ in patients with active sacroiliitis. The finding lacked specificity as vascularization was also found in healthy controls and in patients with osteoarthritis. The resistive index (RI) of the vessels detected by CDUS was significantly lower in patients with sacroiliitis and it increased to the level of the one of controls after treatment.

Several later studies also used Doppler US to detect vascularization in the region of the dorsal SIJ as a sign of inflammation. Although universally they showed an increased vascularization in sacroiliitis, one had to bear in mind the fact that vessels here were also commonly found in healthy subjects. For example, Pakkafahli et al reported vascularization near the dorsal part of the sacroiliac joint, caused by branches of the sacral arteries, in 13 out of 23 healthy controls [27] and Klauser at al found vascularization in 12 out of 21 healthy controls [25]. Furthermore, in a Doppler US study, McGrath et al used vascular signature of the dorsal sacral arteries to identify dorsal sacral nerve rami beneath the posterior long sacroiliac ligament in healthy individuals [28]. The vessels accompanying nerves could be identified in $62 \%$ of the subjects, again in correlation with the above figures.

Unlu et al used CDUS to assess not only the SIJs, but also the thoracic and lumbar paravertebral regions in 39 active Ankylosing spondylitis (AS) patients and 14 controls [29], placing the transducer in the transverse plane over the SIJ and then successively at each spinal level. In patients with active AS, the RI values were significantly lower for all three regions compared to controls. In the subset of patients who underwent anti-TNF therapy, the values of the RI significantly increased for the lumbar region and the SIJs. This finding was confirmed later by Jiang et al, who used power Doppler (PDUS) to assess the SIJs of 55 AS patients, before and after a three-month treatment with Infliximab, and measured the RI of the vessels [30]. They found that compared to baseline, fewer SIJs exhibited blood flow signals after the treatment.

$\mathrm{Hu}$ et al performed a larger study by Doppler US on the SIJs and the major entheses in the lower limbs of 161 patients with AS, dividing them in an active and inactive group [31]. In the active group (Bath Ankylosing Spondylitis Disease Activity Index (BASDAI) value more than 4.0) $90.7 \%$ of patients had vascularization around the dorsal SIJs, compared to $38.5 \%$ in the inactive group (with BASDAI less than 4.0), RI in the active group being significantly lower. There was a good agreement between Doppler US of the SIJs and BASDAI values, while the agreement between Doppler US of the entheses and BASDAI was poor.

The vascularity pattern of the dorsal SIJs was investigated further by Zhu et al using CDUS in 68 patients with AS and 35 controls [32]. They described the occurrence of three different spectral Doppler patterns, the 
one indicative of a venous flow being significantly more common in AS patients with active sacroiliitis. However, in most cases two or more flow patterns were simultaneously observed making CDUS findings' interpretation more difficult.

Finally, Ghosh et al conducted a study that combined $\mathrm{CD}$ and grey scale US of the sacroiliac joints in 29 patients with inflammatory LBP and normal X-ray and 32 controls, comparing the findings with MRI [33]. They found that the observation of three or more flow signals on CDUS and a RI below 0.605 correlated well with the MRI proven cases of sacroiliitis. As far as sensitivity and specificity were concerned, hyperechogenicity of the joint space was the most specific parameter $(95 \%)$, while the most sensitive one was low RI value (94.7\%). Although the study compared nonradiographic sacroiliitis on US to MRI and proposed cutoff values for the first time, it had the limitation of the small sample size, the subjectivity of counting flow signals and the quality of the equipment.

In all the mentioned studies the RI of the vessels was found to be lower in active sacroiliitis and to increase with treatment. However, it is worth noticing that McGrath et al in their study of healthy volunteers found that the RI increased significantly with each caudal sacral level (i.e. from S1 to S3) [28]. Thus, the exact sacral level at which the measurement of RI was taken might be of importance, while none of the studies above provided such data.

\section{US and the soft tissues in the lumbar and sacral regions}

\section{The thoracolumbar fascia}

The thoracolumbar fascia is especially strong in the lower lumbar region, where it envelopes the muscles of the back and is continuous with the gluteal fascia [34]. Its posterior layer has a composite structure and its alteration in patients with chronic LBP was studied sonographically by Langevin et al [35]. They evaluated 60 patients with LBP and 47 controls in a standardized manner, placing a $10 \mathrm{MHz}$ linear transducer in the longitudinal plane on a point $2 \mathrm{~cm}$ lateral to the midpoint at L2-L3 level. They measured the thickness and echogenicity of the "perimuscular zone". This zone was defined as an echogenic layered structure located closest to the muscles' dorsal surface and separated from the nearest, more superficial echogenic layer, by more than $2 \mathrm{~mm}$. In subjects with chronic LBP this zone was found to be $25 \%$ thicker and with greater echogenicity, compared to controls after adjusting for BMI. However, they could not explain if that was the cause or the result of the chronic LBP.
Moreover, the structure under investigation in this study was not well anatomically defined, as the "perimuscular zone" at L2-L3 level would have included tendinous or aponeurotic parts from different muscle groups, as well as perifascial fat tissue and the fascia itself.

The authors partly addressed this issue in a later study [36] where they divided the "perimuscular zone" in two hyperechoic layers made of fibers originating from different muscles and separated by an echolucent layer. They used ultrasound elastography to evaluate tissue displacement during passive trunk motions on an articulated couch and calculated the shear strain stress. They found that the shear strain of the thoracolumbar fascia was about $20 \%$ less in subjects with chronic (more than 12 months) LBP than in controls. Also, overall males had significantly lower shear strain than females, and only in males there was a correlation between the thoracolumbar fascia shear strain and the thickness and echogenicity of the "perimuscular zone". Mean thicknesses of the "perimuscular zone" were $0.49 \mathrm{~mm}$ and $0.41 \mathrm{~mm}$ respectively for the male and female patients with LBP and $0.37 \mathrm{~mm}$ and $0.41 \mathrm{~mm}$ for the male and female control subjects.

\section{The muscles of the lower back and pelvis}

There is a considerable number of papers concerning the so-called rehabilitative ultrasound imaging (RUSI), in which US is used to measure the size (and sometimes to evaluate the structure and contractions) of the paravertebral lumbar muscles [37]. As this technique is not exactly diagnostic and has been extensively reviewed elsewhere [38], it will be mentioned here only briefly. In RUSI, patients are examined in prone position with the transducer placed in a transverse plane over the spinous process and moved laterally to each paravertebral side [39]. Both low frequency linear and curvilinear transducers could be used [40]. The muscle dimensions, shape, and size were taken into consideration and the cross-sectional area of the paraspinal muscles was calculated. The shape and the size of the muscles were similar in healthy individuals of both sexes, and the between-side differences were found to be below $10 \%$ [37]. The extent of the lumbar muscles' atrophy associated with LBP, side to side differences, as well as the muscle group predominantly affected could be well studied by RUSI [37].

The Piriformis syndrome is among the etiologies that are less frequent in LBP, thought to be caused by the thickening and contracture of the piriformis muscle [41]. Our group performed a pilot study on the sonographic features of the Piriformis syndrome using a $7.5-10 \mathrm{MHz}$ linear transducer placed longitudinally to the axis of the muscle [42]. We found out a significant between-side difference in the piriformis muscle's thickness in patients with pain, compared to the control subjects where there 
was a marked symmetry between the left and the right muscles.

\section{The ligaments of the lower back and pelvis}

The posterior sacroiliac ligament (PSL) might be among the LBP generators particularly in pregnancy related posterior pelvic pain [43]. First, Moore et al did an anatomical US study of PSL's long part in cadavers and in healthy young women [44]. Subjects were examined in lateral decubital position using linear $7.5-10 \mathrm{MHz}$ transducer. The ligament was continuously visualized as a hyperechoic fibrillar structure between the posterior iliac spine and the lateral aspect of the third sacral transverse process. Its mean length was $37.9 \pm 2.4 \mathrm{~mm}$, its mean thickness was $1.57 \pm 0.38 \mathrm{~mm}$, and the median angle to the posterior superior iliac spine: $18.5^{\circ}$. There was also a good overall agreement between the PSL length and the thickness in cadavers measured by US and at the dissection.

In 2011, Le Goff et al performed another ultrasound study of the PSL in 20 middle-aged volunteers of both sexes using 5-12 MHz transducer [45]. They separately assessed the short and the long portion of the ligament. The median length of the long part was $34.2 \mathrm{~mm}$ on the left and $35.6 \mathrm{~mm}$ on the right. The ligament was assessed in prone position and was visualized as a fibrillar structure between the inferior part of the posterior superior iliac spine (PSIS) and the lateral side of the third sacral vertebra. Assessment of the short portion of the PSL required more transverse positioning of the probe directly over the sacroiliac joint. It was seen as a fibrillar structure attached to the posterior tuberosity of the ilium and to the sacrum covered by the multifidus muscles. Its median length was $23.1 \mathrm{~mm}$ and $21.7 \mathrm{~mm}$ on the left and right respectively. Dorsal sacral nerve rami normally pass through the space beneath the PSL [29]. As their entrapment in this space was hypothesized to be among the causes for LBP [45], the authors also measured the average area between the ligament and the underlying sacrum $\left(1.19 \mathrm{~cm}^{2}\right.$ on the right, $1.33 \mathrm{~cm}^{2}$ on the left).

Another important ligament of the lower back, namely the iliolumbar ligament (ILL), was studied by Wolenski at al [46]. The authors measured the ligament's thickness in 41 patients and 43 control subjects by US at a point $0.5 \mathrm{~cm}$ above the iliac crest, with the transducer placed in the oblique plane. The median thickness was $3.4 \mathrm{~mm}$ in patients' group and $2.2 \mathrm{~mm}$ in controls' group and the difference was found to be significant. However, as in the above-mentioned studies on the thoracolumbar fascia, it remained controversial whether the increased thickness of the ligament was a cause or an effect of the LBP.

Thus, the main clinical impact of these studies is that they provide good normative data for possible future US assessment of pathologies in the PSL and ILL in patients with LBP.

The posterior ligament complex (PLC) includes the supraspinous and interspinous ligaments, the ligamentum flavum, and the facet joint capsules. In a recent review Alcalá-Cerra et al evaluated the results of four studies on the diagnostic capability of US to detect injuries of these structures in concomitant vertebral fractures [47]. The following US features were considered as pathological: the presence of any disruption of the first echogenic layer; the disruption of the continuous hyperechoic line between spinous processes; the detection of hypoechoic cysts (hematoma); the inhomogeneous arrangement of the ligaments' and muscles' fibers; the identification of avulsed bony fragments. The estimation of the diagnostic accuracy of US using MRI as a gold standard yielded the following results: sensitivity $-90 \%$; specificity $-79 \%$; positive likelihood ratio - 3.63; negative likelihood ratio -0.18 ; and diagnostic odds ratio -24.24 . The authors concluded that US is a highly accurate imaging modality in PLC injuries. However, in most of the studies only the interspinous and supraspinous ligaments were assessed, so the good diagnostic performance of US should be considered only for injuries of these structures and not for lesions of the ligamentum flavum and the facet joint capsules [47].

\section{Iliac crest pain syndrome}

Enthesopathies/tendinopathies cause a considerable number of regional pain syndromes such as Great Trochanter Pain Syndrome or Tennis Elbow [48], but their role as pain generators in LBP has not been established. Our group evaluated by US the iliac entheses of the erector spine (ES) muscle in 60 LBP patients and 50 controls [49]. All patients had clinical signs indicative of LBP caused by a unilateral Iliac Crest Pain Syndrome (ICPS) - a regional pain syndrome described by Collee et al [50]. ICPS is a frequent cause for LBP, its etiology remains obscure and its location coincides with the ES attachment site [5]. In our study, we used linear 10-12 $\mathrm{MHz}$ transducer placed in the oblique plane parallel and perpendicular to the terminal ES tendon. The entheses at the painful side were significantly thicker when compared to both the nonpainful entheses in the patients, and to the same structures in the control subjects. Moreover, painful entheses had significantly more elementary sonopathological lesions (as defined by Terslev et al [51]): hypoechogenicity, altered fibrillar structure, cortical irregularities, and hyperechoic foci than the entheses in both control groups. Our team concluded from this pilot study that enthesopathy of ES could be a discrete cause for LBP (and the underlying lesion of ICPS), identifiable by US. 


\section{Conclusions}

LBP is clearly a complex, multifactorial condition and identifying its most probable cause in any patient requires a vast armamentarium of imaging and clinical methods. At present the number of studies on the diagnostic capability of US in LBP is relatively low, compared to the conventional modalities, and does not allow the drawing of definite conclusions. Nevertheless, the numerous advantages of US such as the low cost, the lack of radiation exposure, the excellent visualization of the soft tissues, makes it more likely that US will be increasingly used by physicians who will examine or treat LBP patients. For this reason, more studies are required, and the American Institute of Ultrasound in Medicine (AIUM) "urges investigators to perform properly designed research projects to evaluate the efficacy of diagnostic spinal ultrasound examinations" [52]. At the same time, AIUM states that currently the use of spinal/paraspinal US in adults for diagnostic or screening evaluations should be considered only investigational.

\section{Conflict of interest: none}

\section{References}

1. Borg-Stein J, Wilkins AN. Soft tissues determinants of low back pain. Curr Pain Headache Rep 2006;10:339-344.

2. Patel ND, Broderick DF, Burns J et al. ACR Appropriateness Criteria Low Back Pain. J Am Coll Radiol 2016;13:10691078.

3. Naredo E. Ultrasound in rheumatology: two decades of rapid development and evolving implementation. Med U1trason 2015;17:3-4.

4. Ruta S, Reginato AM, Pineda C, Gutierrez M. General applications of ultrasound in rheumatology: why we need it in our daily practice. J Clin Rheumatol 2015;21:133-143.

5. Bogduk N. Clinical anatomy of the lumbar spine and sacrum. Elsevier, 2005.

6. Porter RW, Wicks M, Ottewell D. Measurement of the spinal canal by diagnostic ultrasound. J Bone Joint Surg Br 1978;60-B:481-484.

7. Porter RW, Hibbert C, Wellman P. Backache and the lumber spinal canal. Spine (Phila Pa 1976) 1980;5:99-105.

8. Anderson DJ, Adcock DF, Chovil AC, Farrell JJ. Ultrasound lumbar canal measurement in hospital employees with back pain. Br J Ind Med 1988;45:552-555.

9. Macdonald EB, Porter R, Hibbert C, Hart J. The relationship between spinal canal diameter and back pain in coal miners. Ultrasonic measurement as a screening test? J Occup Med 1984;26:23-28.

10. Macdonald EB. Ultrasonic measurement of the lumbar spinal canal. J Occup Med 1984;26:412.
11. Howie DW, Chatterton BE, Hone MR. Failure of ultrasound in the investigation of sciatica. J Bone Joint Surg Br 1983;65:144-147.

12. Tervonen O, Koivukangas J. Transabdominal ultrasound measurement of the lumbar spinal canal. Its value for evaluation of lumbar spinal stenosis. Spine (Phila Pa 1976) 1989;14:232-235.

13. Porter RW, Bewley B. A ten-year prospective study of vertebral canal size as a predictor of back pain. Spine (Phila Pa 1976) 1994;19:173-175.

14. Merx JL, Thijssen HO, Meyer E, Chung RW. Accuracy of ultrasonic evaluation of lumbar intervertebral discs by an anterior approach. Neuroradiology 1989;31:386-390.

15. Kamei K, Hanai K, Matsui N. Ultrasonic level diagnosis of lumbar disc herniation. Spine (Phila Pa 1976) 1990;15):1170-1174.

16. Tervonen $\mathrm{O}$, Lähde S, Vanharanta H. Ultrasound diagnosis of lumbar disc degeneration. Comparison with computed tomography/discography. Spine (Phila Pa 1976) 1991;16:951-954.

17. Berth A, Mahlfeld K, Merk HR. Transabdominal ultrasonography in the diagnosis of lumbar disc herniation. U1traschall Med 2003;24:383-387.

18. Buyruk HM, Snijders CJ, Vleeming A, Laméris JS, Holland WP, Stam HJ. The measurements of sacroiliac joint stiffness with colour Doppler imaging: a study on healthy subjects. Eur J Radiol 1995;21:117-1121.

19. Buyruk HM, Stam HJ, Snijders CJ, Laméris JS, Holland WP, Stijnen TH. Measurement of sacroiliac joint stiffness in peripartum pelvic pain patients with Doppler imaging of vibrations (DIV). Eur J Obstet Gynecol Reprod Biol 1999;83:159-163.

20. Damen L, Buyruk HM, Guler-Uysal F, Lotgering FK, Snijders CJ, Stam HJ. Pelvic pain during pregnancy is associated with asymmetric laxity of the sacroiliac joints. Acta Obstet Gynecol Scand 2001;80:1019-1024.

21. Damen L, Buyruk HM, Guler-Uysal F, et al. The prognostic value of asymmetric laxity of the sacroiliac joints in pregnancy-related pelvic pain. Spine (Phila $\mathrm{Pa} 1976$ ) 2002;27:2820-2824.

22. de Groot M, Spoor CW, Snijders CJ. Critical notes on the technique of Doppler imaging of vibrations (DIV). Ultrasound Med Biol 2004;30:363-367.

23. Spadaro A, Iagnocco A, Baccano G, Ceccarelli F, Sabatini E, Valesini G. Sonographic-detected joint effusion compared with physical examination in the assessment of sacroiliac joints in spondyloarthritis. Ann Rheum Dis 2009;68:1559-1563.

24. Klauser A, Halpern EJ, Frauscher F, et al. Inflammatory low back pain: high negative predictive value of contrastenhanced color Doppler ultrasound in the detection of inflamed sacroiliac joints. Arthritis Rheum 2005;53:440444.

25. Klauser AS, De Zordo T, Bellmann-Weiler R, et al. Feasibility of second-generation ultrasound contrast media in the detection of active sacroiliitis. Arthritis Rheum 2009;61:909-916. 
26. Arslan H, Sakarya ME, Adak B, Unal O, Sayarlioglu M. Duplex and color Doppler sonographic findings in active sacroiliitis. AJR Am J Roentgenol 1999;173:677-680.

27. Pekkafahli MZ, Kiralp MZ, Basekim CC, et al. Sacroiliac joint injections performed with sonographic guidance. J Ultrasound Med 2003;22:553-559.

28. McGrath CM, Jeffery R, Stringer MD. The dorsal sacral rami and branches: Sonographic visualization of their vascular signature. Int J Osteopath Med 2011;15:3-12.

29. Unlu E, Pamuk ON, Cakir N. Color and duplex Doppler sonography to detect sacroiliitis and spinal inflammation in ankylosing spondylitis: can this method reveal response to anti-tumor necrosis factor therapy? J Rheumatol 2007;34:110-116.

30. Jiang Y, Chen L, Zhu J, et al. Power Doppler ultrasonography in the evaluation of infliximab treatment for sacroiliitis in patients with ankylosing spondylitis. Rheumatol Int 2013;33:2025-2029.

31. Hu Y, Zhu J, Xue Q, Wang N, Hu B. Scanning of the sacroiliac joint and entheses by color Doppler ultrasonography in patients with ankylosing spondylitis. J Rheumatol 2011;38:1651-1655.

32. Zhu J, Xing C, Jiang Y, Hu Y, Hu B, Wang N. Evaluation of complex appearance in vascularity of sacroiliac joint in ankylosing spondylitis by color Doppler ultrasonography. Rheumatol Int 2012;32:69-72.

33. Ghosh A, Mondal S, Sinha D, Nag A, Chakraborty S. Ultrasonography as a useful modality for documenting sacroiliitis in radiographically negative inflammatory back pain: a comparative evaluation with MRI. Rheumatology (Oxford) 2014;53:2030-2034.

34. Benjamin M. The fascia of the limbs and back - a review. J Anat 2009;214:1-18.

35. Langevin HM, Stevens-Tuttle D, Fox JR, et al. Ultrasound evidence of altered lumbar connective tissue structure in human subjects with chronic low back pain. BMC Musculoskelet Disord 2009;10:151.

36. Langevin HM, Fox JR, Koptiuch C, et al. Reduced thoracolumbar fascia shear strain in human chronic low back pain. BMC Musculoskelet Disord 2011;12:203.

37. Stokes M, Hides J, Elliott J, Kiesel K, Hodges P. Rehabilitative ultrasound imaging of the posterior paraspinal muscles. J Orthop Sports Phys Ther 2007;37:581-595.

38. Heidari P, Farahbakhsh F, Rostami M, Noormohammadpour P, Kordi R. The role of ultrasound in diagnosis of the causes of low back pain: a review of the literature. Asian J Sports Med 2015;6:e23803.

39. Hides JA, Cooper DH, Stokes MJ. Diagnostic ultrasound imaging for measurement of the lumbar multifidus mus- cle in normal young adults. Physiother Theory and Pract 1992;8:19-26.

40. Whittaker JL, Teyhen DS, Elliott JM, et al. Rehabilitative ultrasound imaging: understanding technology and its applications. J Orthop Sports Phys Ther 2007;37:434-449.

41. Hopayian K, Song F, Riera R, Sambandan S. The clinical features of the piriformis syndrome: a systematic review. Eur Spine J 2010;19:2095-2109.

42. Todorov P, Nestorva R, Batalov A. Sonographic features of the piriformis syndrome. Ann Rheum Dis 2014;73(Suppl 2):296-297.

43. Vleeming A, Mooney V, Stoeckart R. Movement, stability and lumbopelvic pain. Integration of research and therapy. Churchill Livingstone Elsevier, 2007.

44. Moore AE, Jeffery R, Gray A, Stringer MD. An anatomical ultrasound study of the long posterior sacroiliac ligament. Clin Anat 2010;23:971-977.

45. Le Goff B, Berthelot J-M, Maugars Y. Ultrasound assessment of the posterior sacroiliac ligaments. Clin Exp Rheumatol 2011;29:1014-1017.

46. Wolenski L, Bizzi E, Galletti S, Galletti R, Marchi A. Diagnostic classification of ileum-lumbar ligamentite. Ann Rheum Dis 2015;74(Suppl 2):1255.

47. Alcalá-Cerra G, Paternina-Caicedo Á, Gutiérrez-Paternina J, Moscote-Salazar L, Alvis-Miranda H, Sabogal-Barrios R. Diagnostic accuracy of ultrasound for detecting posterior ligamentous complex injuries of the thoracic and lumbar spine: A systematic review and meta-analysis. J Craniveretbral Junction Spine 2013;4:25-31.

48. Benjamin M, Toumi H, Ralphs JR, Bydder G, Best TM, Milz S. Where tendons and ligaments meet bone: attachment sites ('entheses') in relation to exercise and/or mechanical load. J Anat 2006;208:471-490.

49. Todorov P, Nestorova R, Batalov A. Use of musculoskeletal ultrasound to detect painful enthesopathies in low back pain. Proceedings of the 9th Intradisciplinary World Congress on Low Back and Pelvic Girdle Pain, Singapore, 2016:220-223.

50. Collee G, Dijkmans BA, Vandenbrucke JP, Cats A. Iliac crest pain syndrome in low back pain: frequency and features. J Rheumatol 1991;18:1064-1067.

51. Terslev L, Naredo E, Iagnocco A, et al. Defining enthesitis in spondyloarthritis by ultrasound: results of a Delphi process and of a reliability reading exercise. Arthritis Care Res (Hoboken) 2014;66:741-748.

52. The American Institute for Ultrasound in Medicine. Official Statement: Nonoperative spinal/paraspinal ultrasound in adults. April 2, 2014, http://www.aium.org/officialStatements/18, accessed April 24, 2017. 